Document downloaded from:

http://hdl.handle.net/10251/99228

This paper must be cited as:

Franco Pereyra, RD.; Ortiz Bas, A.; Gómez-Gasquet, P. (2013). Service-oriented Approach Supporting Dynamic Manufacturing Networks Operations. IFIP Advances in Information and Communication Technology. 408:345-354. doi:10.1007/978-3-642-40543-3_37

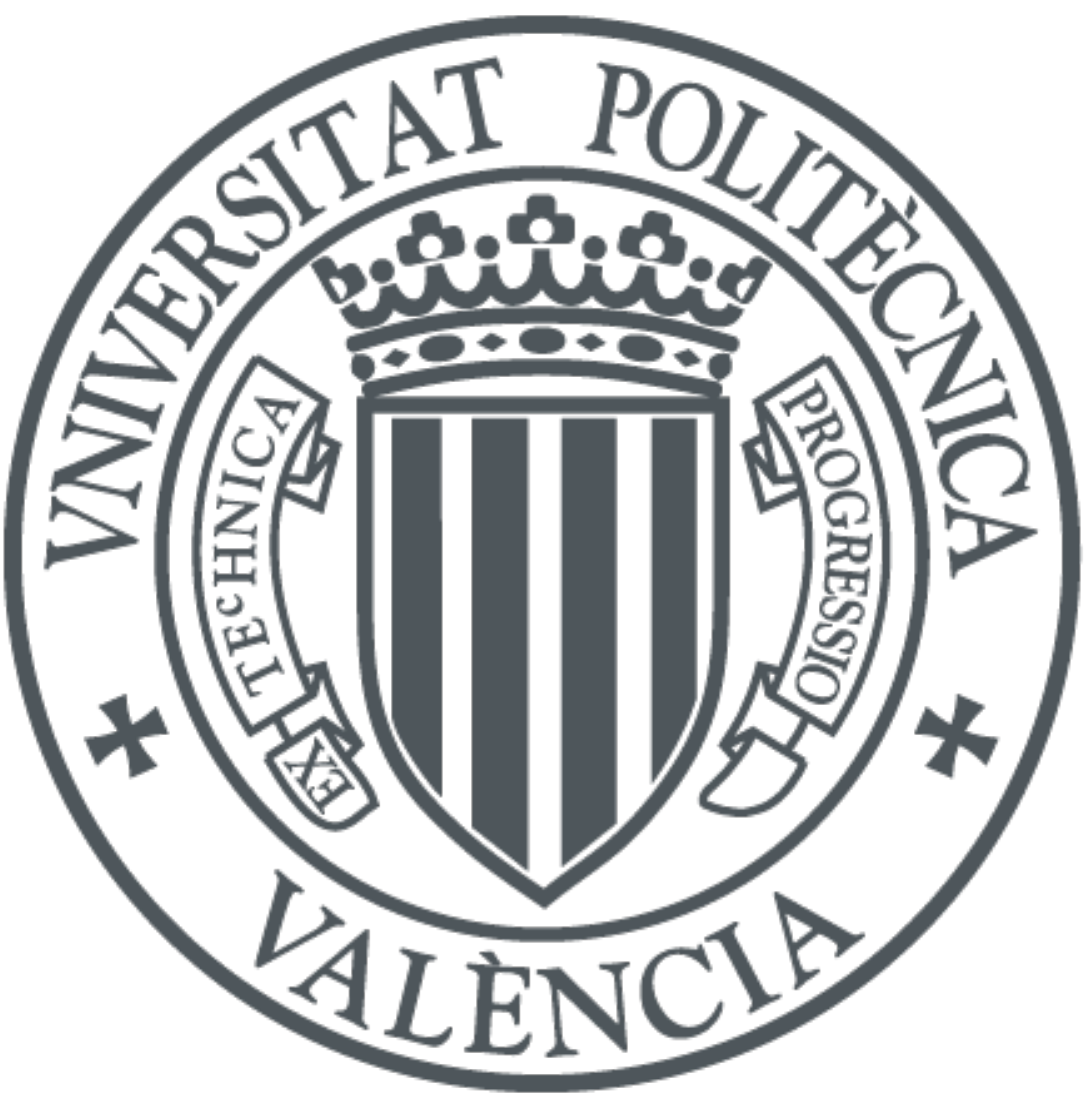

The final publication is available at

https://doi.org/10.1007/978-3-642-40543-3_37

Copyright Springer

Additional Information 


\title{
Service-oriented Approach Supporting Dynamic Manufacturing Networks Operations
}

\author{
Rubén Darío Franco Pereyra ${ }^{1}$, Ángel Ortiz Bas ${ }^{1}$, Pedro Gómez Gasquet ${ }^{1}$ \\ ${ }^{1}$ Universitat Politécnica de Valencia \\ Research Centre on Production Managemen and Enginering, \\ 46022 Valencia, Spain \\ \{dfranco, aortiz,pgomez\}@cigip.upv.es
}

\begin{abstract}
In the current economic crisis, also the manufacturing sector is asked to evolve towards more dynamic organizational structures within which, composing manufacturing processes, almost in real time, will become a need. This work aims at introducing flexibility and dynamisms to current manufacturing processes by separating its tasks from its final performers. With the proposed approach, the performers' replacement can be done almost seamlessly. Additionally, the approach shows how dynamic negotiation and contracting, either for a whole process or a single activity, can be smoother if the task specification is based on a standard service interface defined at the ecosystem level. At the end, a prototype implementation is briefly described.
\end{abstract}

Keywords: Dynamic Manufacturing Networks, Service-Orientation, Crossorganizational Process Management.

\section{Introduction}

In terms of collaboration and dynamism, traditional manufacturing networks have been mostly characterized by stable and permanent relationships among its members. Changes in the network structure were not easy to find and a tight coupling between a required task and the expected performer was a predominant model.

However, perspectives on current economic situation require a more agile assembly, disassembly and re-assembly of manufacturing networks, by combining a set of specific capabilities being offered to third parties, almost 'on the fly'. New mechanisms for setting up and running more efficient and responsive organizational structures are needed. Focused on how to better synchronize their networked operations, these new organizational structures would also be able to provide:

- Enhanced organizational permeability: That is to make available more efficient mechanisms for dynamic partners' selection, almost 'on the fly'.

- Extend the potential performers' base. Opening the networks to a higher potential number of members. For SMEs, a source of business opportunities.

- "Plug \& play" business interfaces. Understanding a business interface as the operational linkage that enables the connection of a company to an existing 
network, the design and implementation of such interfaces is aimed at being focused on interoperability for achieving greater flexibility.

Based on these requirements, this work proposes an approach aimed at supporting the operations of Dynamic Manufacturing Networks (DNM). Such dynamism is expected to be achieved by selecting business partners and contracting their services at runtime, as a way of realizing the manufacturing process executions.

Thus, traditional modeling and execution of manufacturing processes is complemented with a service-oriented architecture design (SOA).

The rest of the paper is structured as follows: Section 2 introduces some background concepts which are used to assemble the proposal. Section 3 provides an insight on DNM, their context, operational requirements and the application of the Service Entities approach for process modeling and execution. Sections 4 and 5 briefly introduce the architecture and implementation of a software prototype.

\section{Related Concepts}

The overall framework inside which this work is developed, takes advantage of the synergies already described in the literature between Open Ecosystems [1], as manifestations of Virtual Organizations Breeding Environments (VBE) [2], [8], [9], [10], and Collaborative Networked Organizations (CNO) [2]. More specifically, this work may be considered a particularization of the framework proposed in [3].

Besides there exist several perspectives from which such synergies can be described, to the end of this work, authors have focused on the operational one, paying special attention to the role played by cross-organizational business processes and the performers of tasks as in [4] and [5].

In second place, this work adopts the Switching Principle introduced by Mowshowitz [6] who considers that it lies at the heart of the management of dynamic virtual organizations. According to the author, its main considerations are:

"Logical separation of need from need-fulfillment is the foundation of virtual organization. If a task or activity is managed by treating need and need-fulfillment independently, it is possible to think about switching or changing the assignment of need-fulfillment to need in a systematic way..."

Finally, this work also explores the role of Service Oriented Architecture (SOA) in supporting the design of interoperable business interfaces, being able to realize the switching principle application. The Service Entities approach [7] is also used as main enabler of the whole architecture.

\section{Dynamic Manufacturing Processes}

Next sections will describe a proposal to provide dynamic features to traditional manufacturing processes. Such dynamism will be guided by the above described Switching Principle and it is expected those processes can be more flexible at both modeling and execution stages. 


\subsection{Manufacturing Ecosystems and Industrial Manufacturing Networks}

This work focuses on a particular type of VBE manifestation, related to manufacturing activities called Manufacturing or Industrial Ecosystems [11].

Ecosystems are populated by unique individuals that belong to one of the species existing inside it. In turn, those species can be mostly described in terms of their distinguishing attributes and behavioral features. By nature, ecosystems are open spaces where new individuals have almost no restrictions to come in or leave it.

When translated to an industrial context, the significance of the term can be applied to all the possible resources that can be arranged together in order to produce goods or services that some end customer is demanding for. Such resources are also unique; belong to some 'specie' (i.e. they have a set of distinguishing attributes and a functional profile) and they may join or leave different business environment according to their strategies. When arranged in a new organizational structure, an industrial manufacturing network is created and ready to operate.

Despite there may exist many particular species inside the manufacturing ecosystem, some originating classification of them can be made: productive resources (with transforming capabilities); warehouse resources (they store produced materials or components); and transportation resources (moving goods among nodes).

\subsection{Service Entities in Manufacturing Ecosystems}

In past contributions [12], [13], [14], Service Oriented Architectures (SOA) have been proposed as framework for service-centric systems development in manufacturing contexts.

Besides this work also considers SOA a fundamental construct for the proposal, the Service Entities (SE) approach is adopted. SE are used for creating service-based profiles of companies that, later on, will be part of a service ecosystem.

Two different types of SE can be identified. Abstract Service Entities (ASE) refers to generic profiles of species populating the ecosystem. Those profiles are created by defining a set of attributes common to all the individuals and a set of service interfaces which will constitute their behavioral side (functional capabilities). Concrete Service Entities will be created by instantiating ASE, by selecting the corresponding profile, valuing attributes and implementations abstract interfaces.

When applied to manufacturing ecosystems, three main types of entities can be found (see Fig. 1):

- Productive Entities: entities in charge of transforming raw materials or semifinished goods into a new component or finished good. Plants, factories, assemblies, lines, cells, machines, or even robots can be considered entities in this category. $\mathrm{ASE}_{\text {Productive }}=\{$ "Central plant", "Subcontractor", ... $\}$

- Warehouse Entities: these entities store both finished and semi-finished products along the network. They have not transformation capabilities and may be present along the whole network. Central warehouses or retailers are examples of this kind of entities. $\mathrm{ASE}_{\text {Warehouse }}=\{$ "Central Warehouse", "Distribution center", "Retailer",... $\}$ 
- Transport Entities: entities are in charge of moving goods from any possible combination of the two aforementioned entities. In this case, two examples may be: $\mathrm{ASE}_{\text {Transport }}=\{$ "LogisticOperator", "InternalTransport" $\}$

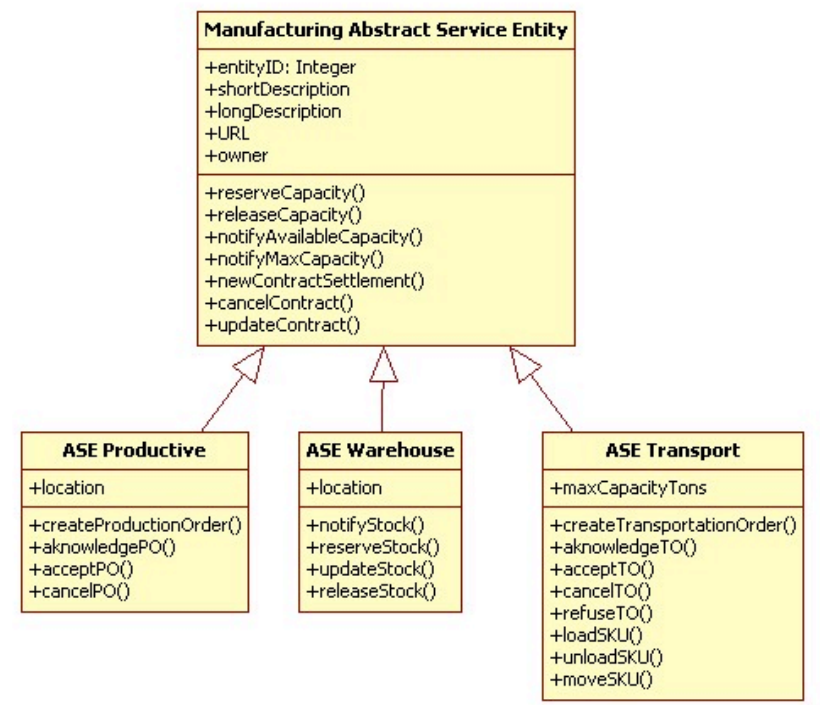

Fig. 1. Abstract Service Entities for Manufacturing Ecosystems.

As previously mentioned, each ASE has a set of harmonized service interfaces, $\mathrm{S}=$ $\{\mathrm{s} 1, \mathrm{~s} 2, \ldots, \mathrm{sn}\}$, which may encompass different business activities (or even whole processes). These interfaces are defined generically for the ASEs and they will be implemented for each CSE, depending on each node's requirements.

For the ASE identified above, some illustrative services may include (see Table 1):

Table 1. Service interfaces for different Manufacturing ASE.

\begin{tabular}{|c|c|c|c|}
\hline \multirow[t]{2}{*}{ Service name } & \multicolumn{3}{|c|}{ ASE } \\
\hline & Productive & Warehouse & Transport \\
\hline reserveCapacity() & $\bullet$ & $\bullet$ & $\bullet$ \\
\hline releaseCapacity() & $\bullet$ & - & - \\
\hline notifyAvailableCapacity() & - & $\bullet$ & - \\
\hline notifyMaxCapacity () & $\bullet$ & - & - \\
\hline createProductionorder() & $\bullet$ & & \\
\hline aknowledgeProductionorder() & $\bullet$ & & \\
\hline cancelProductionorder() & $\bullet$ & & \\
\hline notifystock() & & $\bullet$ & \\
\hline updatestock () & & • & \\
\hline createTransportationorder () & & & $\bullet$ \\
\hline loadSKU ( ) & & & • \\
\hline moveSKU ( ) & & & - \\
\hline
\end{tabular}


The above list is not exhaustive. It must be considered as illustrative and it may vary according to specific modeling needs of each particular manufacturing ecosystem.

Service interfaces are defined by the ecosystem manager and they must be preserved when each concrete node performs their local implementations. Hence, a decoupling point is established here: all the ecosystem entities belonging to the same ASE profile will share the same set of interfaces but their local implementations will be done according to their specific needs.

Thus, ASE specifications will be acting in a similar way than, for example, a printer device driver does. A device driver provides the linkage between the physical devices and the operating system by implementing the set of functions that will be invoked when the OS needs some service from it. Each printer manufacturer performs their implementation of those functions according to each specific device.

\subsection{Process modeling with Service Entities}

As it was stated in the introduction, this approach establishes a clear separation between process activities and their performers for both its modeling and execution.

From a structural perspective, network dynamicity is expected to be achieved by enabling a seamless replacement among performers, in a cost-effective way. SE approach comes to support this requirement by allowing to select one performer among the whole set of CSE belonging to the same ASE profile.

The functional dynamism will be supported by enabling the selection of an individual performer for either a whole process or a single task for a single process instance. That means, from the SE approach, to select a CSE to carry out a process activity but only for this concrete process instance. Future instances may be assigned to another CSE sharing the same ASE profile.

Depending on the expected dynamism, the performers selection and the number of activities they will be requested to provide, three different cases are identified:

- Static Process Contracting: Performers are known and selected at process design time and that will be involved in all its future instances. They will carry out all the activities expected for each specific profile.

- Dynamic Process-level Contracting: Performers will be selected at runtime, for 'this' specific process instance. Dynamic process bidding will not ensure future involvements for them. They are contracted to carry out all the activities of the process instance.

- Dynamic Service-level Contracting: Performers that will be requested to provide a single service, for a concrete instance of a process. At different process steps (activities) they will be competing against all those similar nodes belonging to the same profile.

Next, how the SE approach can be used to support these requirements (see Fig. 2). 


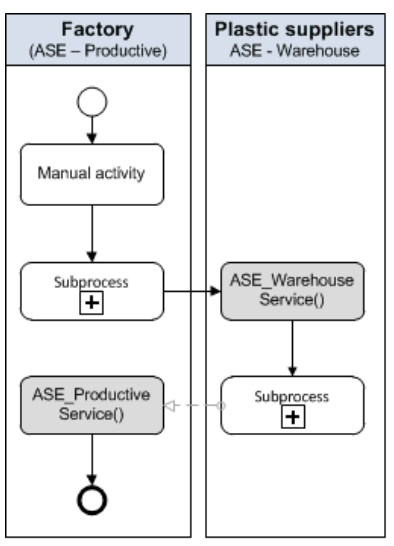

1. Reference Process

Actors: Only Abstract Service

Entities can be used for process modeling

Activities: Include abstract service interfaces

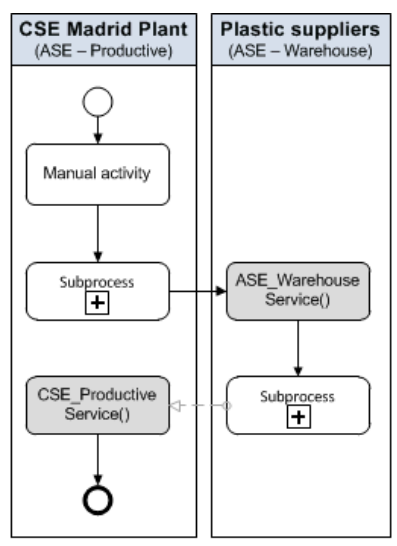

\section{Dynamic Process}

Actors: Concrete Service Entities but also ASE may be used. Task assignments are solved dinamycally

Activities: Implemented services of CSE must be used. ASE services are bounded dinamically

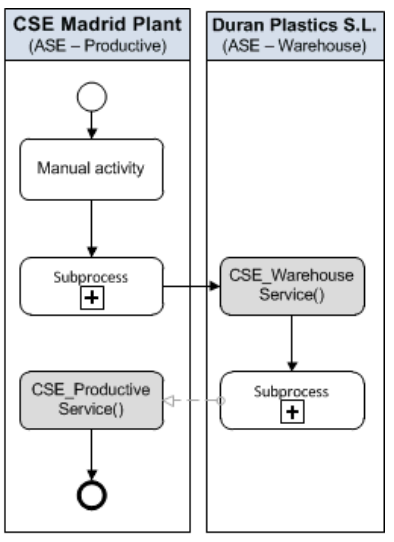

\section{Concrete Process}

Actors: Only Concrete Service

Entities Task assignments are solved at design time.

Activities: Implemented services of CSE are used.

Fig. 2. Different process types modeled with SE.

In modeling such heterogeneity of dynamic manufacturing processes with SE, three different templates are supported:

- Reference Process: a reference process template is the one that is created only from ASE profiles. The process activities may include ASE services and performers are not specified.

- Dynamic Process: a dynamic process represents a combination of static and dynamically selected performers. Contracted performers may be asked to provide a single service or all the activities of a process. ASE interfaces will be used as task specification for negotiation, contracting and assignment.

- Concrete Process: this template is used to support static processes. Performers and task assignments remain unaltered at design and execution time. Process activities may include concrete service implementations.

\subsection{Dynamic Manufacturing Process Models}

As introduced in the previous section, SE approach provides different templates for process modeling. In Figure 3, how the Dynamic Process template is used to create a process model involving several actors is depicted.

The example illustrates how a concrete actor (Operations Planning Dept) is going to manage the incoming orders from a productive resource of its supply network. To create the process model, no additional concrete performers have been specified. Only a productive and a transport ASE profiles were introduced. The highlight (1) of the figure shows how service interfaces have been used to create the process model. 
The highlight (2) of the figure introduces an additional element in dealing with the process dynamism. Since no CSE has been selected at design time, this may have to different implications, depending on each process specific requirements:

- A single performer will be decided at runtime and will provide the requested services to realize the process instance.

- At runtime, all the CSE belonging to the selected profile will be queried about, for instance, current availability of some component and further decisions will be taken based on the replies.

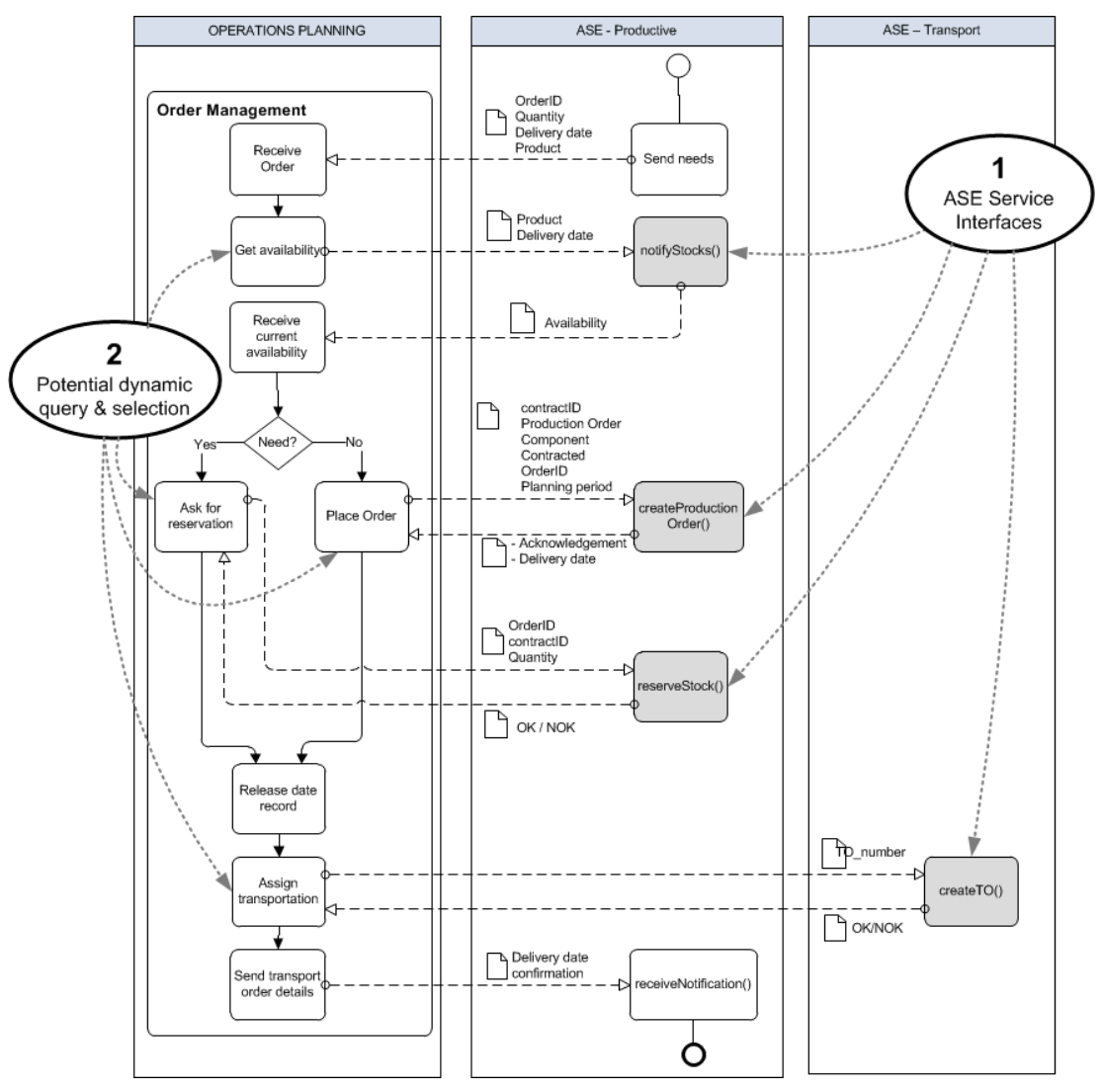

Fig. 3. Dynamic manufacturing process model including SE.

\section{Prototype implementation}

Next sections describe a prototype implementation of an IT platform aimed at supporting modeling and execution of dynamic manufacturing processes. 


\subsection{Platform architecture}

The platform architecture consists of four main modules (see Fig. 4):

- ASE modeling module is aimed at supporting the creation of all the different ASE profiles that will populate the manufacturing ecosystem.

- CSE instantiation: this module is in charge of managing the instantiation process of ASE into CSE. The ecosystem repository is fed new instances.

- Process modeling: this module consists of a BPMN process editor that provides manufacturing process templates as described in Section 3.2.

- Orchestration engine: the orchestration engine is in charge of supporting the execution of concrete process instances. The engine must include advanced negotiation capabilities and service-based business rules.

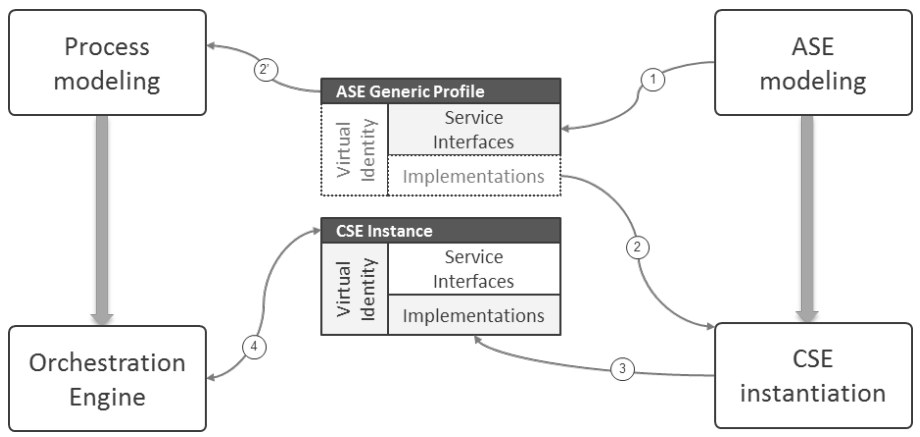

Fig. 4. Simplified architecture of the platform architecture.

The functional integration of these modules aims supports the requirements of DNM. In the Figure 4 arrows schematically represent methodological steps to be followed. In (1), ASE modeling comes out with the ASE profiles which include attributes and service interfaces. Based on the ASE profiles, in (2) attributed are valued and concrete implementations of services are provided by each node. Depending on the process template, in (2') existing models of ASE and CSE can be extracted from the ecosystem repository. In (3) CSE instances become visible inside the ecosystem. From now on they can be asked to be part of a new manufacturing network or be part of an existing one, being asked to provide some specific service of their profiles. Finally, at runtime (4) the orchestrator engine request and consume concrete services of CSE being part of the Manufacturing Ecosystem, at that specific moment in time.

\subsection{Technical implementation}

The technical implementation of the prototype has resulted in a web-based tool that exemplifies typical requirements of a manufacturing network which needs to perform its operations planning for all the network nodes.

The prototype has been developed by using .NET technologies. The programming language is VB.NET and the database supporting the implementation is PostgreSQL. 


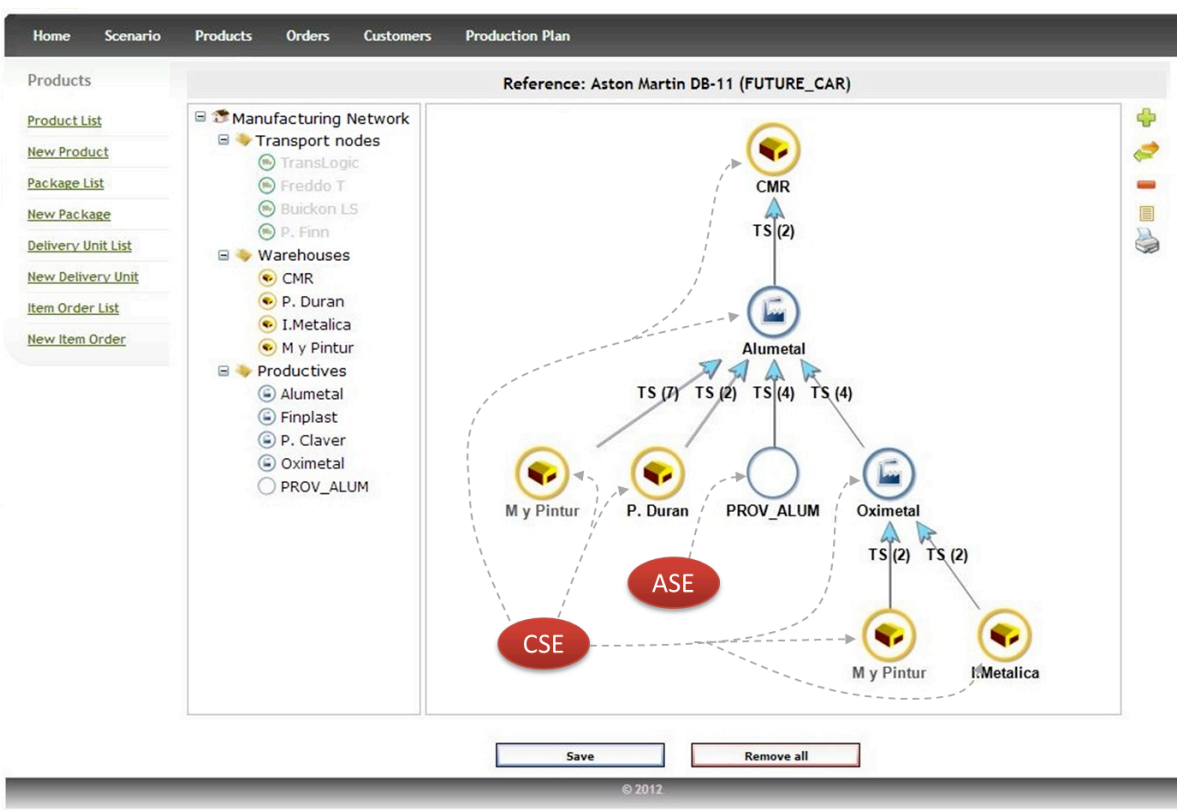

Fig. 5. Screen capture of the implemented prototype.

The screenshot depicted in Fig. 5 shows a particularization done for this specific scenario that consists in creating a visual editor that combines both the product and the process structure definition.

\section{Conclusions and Potential Contribution to Reindustrialization}

The manufacturing sector needs more dynamic organizational structures. The approach introduced here provides flexibility and dynamisms to current manufacturing processes by separating its tasks from its final performers. With the support of a service-centric architectural approach, new requirements have been identified for future process orchestration engines.

By incorporating the SE approach for manufacturing process modeling and execution, the application of the Switching Principle of Virtual Organizations seems to be more achievable. As it has been highlighted, the performers' replacement can be done seamlessly, since the approach ensures the lowest interoperability effort. Additionally, dynamic negotiation and contracting, either for a whole process or a single activity, can be easier if the task specification is based on a standard service interface defined at the ecosystem level.

Concerning how this approach may be helpful in supporting the reindustrialization that this economic crisis is demanding from the manufacturing sector, authors believe that companies will need new approaches and tools that enable them to assemble and disassemble dynamic manufacturing networks with the lowest effort as possible. 
This approach is aimed at providing a piece of the whole solution and perhaps its main contribution to solve the problem may be the permanent focus on an interoperability-based design of the whole approach.

\section{References}

[1] L. Osório, H. Afsarmanesh, and L. M. Camarinha-Matos, "Open Services Ecosystem Supporting Collaborative Networks," in Balanced Automation Systems for Future Manufacturing Networks, vol. 322, A. Ortiz Bas, R. D. Franco, and P. Gómez-Gasquet, Eds. Berlin, Heidelberg: Springer Berlin Heidelberg, 2010, pp. 80-91.

[2] H. Afsarmanesh, L. Camarinha-Matos, and S. Msanjila, "On management of 2nd generation Virtual Organizations Breeding Environments," Annual Reviews in Control, vol. 33, no. 2, pp. 209-219, Dec. 2009.

[3] H. Afsarmanesh and L. Camarinha-Matos, "A Framework for Management of Virtual Organization Breeding Environments," in Collaborative Networks and Their Breeding Environments, 2005, pp. 35-48.

[4] R. Franco, A. Ortiz Bas, P. Gómez-Gasquet, and R. Rodriguez Rodriguez, "Open Ecosystems, Collaborative Networks and Service Entities Integrated Modeling Approach," in Collaborative Networks in the Internet of Services, vol. 380, L. CamarinhaMatos, L. Xu, and H. Afsarmanesh, Eds. Springer Berlin Heidelberg, 2012, pp. 74-83.

[5] J. Minguez, S. Zor, and P. Reimann, "Event-driven business process management in Engineer-to-Order supply chains," in 2011 15th International Conference on Computer Supported Cooperative Work in Design (CSCWD), 2011, pp. 624-631.

[6] X. Wang, H. Cai, and B. Xu, "An Extended Petri-Net Based Approach for Supply Chain Process Modeling and Web Service Transformation," in International Conference on Management and Service Science, 2009. MASS '09, 2009, pp. 1-5.

[7] A. Mowshowitz, "The switching principle in Virtual Organization," eJOV, vol. 1, no. 1, pp. 7-18, 1999.

[8] Franco, Ortiz Bas, and F. C. Lario Esteban, "Modeling extended manufacturing processes with service-oriented entities," Service Business, vol. 3, no. 1, pp. 31-50, Mar. 2009.

[9] R. V. Vallejos, C. Lima, and G. Varvakis, "A Framework To Create A Virtual Organization Breeding Environment In The Mould And Die Sector," in Network-Centric Collaboration and Supporting Frameworks, Springer US, 2006, pp. 599-608.

[10] L. Bocchi, J. Fiadeiro, N. Rajper, and S. Reiff-Marganiec, "Structure and Behaviour of Virtual Organisation Breeding Environments," Jan. 2010.

[11] D. Romero and A. Molina, "VO breeding environments \& virtual organizations integral business process management framework," Information Systems Frontiers, vol. 11, no. 5, pp. 569-597, 2009.

[12] M. Ulieru and S. Grobbelaar, "Engineering Industrial Ecosystems in a Networked World," in 5th IEEE International Conference on Industrial Informatics, 2007, vol. 1, pp. 1-7.

[13] S. N. S. Rajini and D. T. Bhuvaneswari, "Service Based Architecture for Manufacturing Sector," 2010.

[14] A. Lobov, J. Puttonen, V. V. Herrera, R. Andiappan, and J. L. M. Lastra, "Service oriented architecture in developing of loosely-coupled manufacturing systems," in 6th IEEE International Conference on Industrial Informatics, INDIN 2008,, pp. 791-796.

[15] F. Jammes, H. Smit, J. L. M. Lastra, and I. M. Delamer, "Orchestration of serviceoriented manufacturing processes," in 10th IEEE Conference on Emerging Technologies and Factory Automation, 2005. ETFA 2005, 2005, vol. 1, p. 8 pp.-624.

[16] S. Zor, K. Görlach, and F. Leymann, "Using BPMN for Modeling Manufacturing Processes.” Wien, Graz: Neuer Wissenschaftlicher Verlag, May-2010. 